\title{
Undenatured Parvovirus B19 Antigens Are Essential for the Accurate Detection of Parvovirus B19 IgG
}

\author{
S. Kerr, ${ }^{1 *}$ G. O'Keeffe, ${ }^{1}$ C. Kilty, ${ }^{1}$ and S. Doyle ${ }^{2}$ \\ ${ }^{1}$ Biotrin International, The Rise, Mount Merrion, Dublin, Ireland \\ ${ }^{2}$ Department of Biology, National University of Ireland, Maynooth, Kildare, Ireland
}

Recombinant versions of parvovirus B19 capsid proteins VP1 and VP2 are used for immunodiagnostic assays for detection of antiviral antibodies. The immune response to B19 is characterized by a gradual loss of antibodies directed against linear epitopes of VP2. A similar occurrence for antibodies raised against VP1 protein would represent a limitation to serological assays incorporating denatured versions of either viral antigen. Four detection systems for B19 lg detection have been developed, including an IgG enzyme immunoassay (EIA) based on undenatured VP2, an immunofluorescence assay (IFA) based on undenatured VP1, a Western blot assay incorporating denatured VP1 and VP2, and an alternative blot system using denatured VP1 but undenatured VP2. Specimens $(n=108)$ were tested by all four systems and identical results were obtained by EIA, IFA, and alternative blot systems, whereby 75/108 (69\%) were B19 IgGpositive. Twelve B19 lgG-positive specimens, representing $16 \%(12 / 75)$ of the confirmed positives, did not react to either viral antigens when tested by Western blot. It is concluded that these sera do not react with linear epitopes of VP1 and VP2 antigens. Eighty-five different specimens, which had previously been shown to be both B19 IgM- and IgG-positive by EIA and IFA, were positive by B19 IgM and IgG Western blot. In the IgG Western blot assay, 69 reacted with both VP1 and VP2 and 16 with VP1 only. It is concluded that there is a requirement for at least one undenatured antigen for the immunological detection of B19 IgG. J. Med. Virol. 57:179-185, 1999. () 1999 Wiley-Liss, Inc.

KEY WORDS: immunoassay; epitopes; diagnosis; infection; fetus; pregnancy

\section{INTRODUCTION}

Parvovirus B19 is the causative agent of erythema infectiosum in humans [Anderson et al., 1983]. The dis- ease is symptomatically mild in healthy individuals and is most prevalent during childhood. In adults, infection has sometimes been associated with persistent arthritis; in addition, the infection can be lifethreatening to the fetus, immunocompromised patients, and individuals with hematopoetic disorders due to viral replication in erythroid precursor cells [Lefrer et al., 1986; Anand et al., 1987; Kurtzman et al., 1987; Woolf et al., 1989; Serjeant et al., 1993]. Approximately $60-70 \%$ of the adult population is parvovirus B19 IgG-positive [Brown, 1997].

In the mature virion, structural proteins VP2 and VP1 compose $95 \%$ and $5 \%$ of total capsid protein, respectively [Ozawa et al., 1987]. The coding region for both proteins is located in the right-hand section of the viral genome where overlapping genes encode both VP1 and VP2 [Ozawa et al., 1987]. Thus, VP1 protein contains the entire VP2 amino acid sequence plus an additional 227 amino acids. The three-dimensional structure of VP2 capsids alone and VP2 capsidgloboside receptor complex have been reported and it has been suggested that epitopes for neutralizing antibodies map onto the capsid surface at regions immediately surrounding the globoside attachment sites [Chipman et al., 1996].

Immunodiagnostic tests available for parvovirus B19 IgG and IgM detection include enzyme immunoassay (EIA), immunofluorescent assay (IFA), and Western blot. These methods rely on the respective antibody reactivity with either or both of the parvovirus B19 proteins, VP1 (84 kDa) and VP2 (58 kDa). In such tests, the recombinant proteins used are extracted from either $E$. coli or recombinant baculovirus-infected Spodoptera frugiperda cells [Morinet et al., 1989; Brown et al., 1991].

Using denatured VP1- and VP2-based Western blot tests for detecting parvovirus B19 IgG, it has been observed that in the majority of cases reactivity is con-

\footnotetext{
*Correspondence to: Dr. S. Kerr, Biotrin International, The Rise, Mount Merrion, Co. Dublin, Ireland. E-mail: skerr@biotrin.ie

Accepted 29 July 1998
} 
fined to VP1; however, such sera will react with undenatured VP2 when used in an EIA [Söderlund et al., 1995]. This phenomenon has been confirmed in our laboratory, which specializes in parvovirus B19 diagnostic development and manufacture. Furthermore, it has been shown that this nonreactivity toward denatured VP2 occurs after about 6-12 months postinfection, but that prior to this parvovirus B19 IgG will react with linear epitopes of both denatured VP1 and VP2 proteins in Western blots [Franssila et al., 1996].

In our laboratory it was also noted that a number of sera, which gave strongly positive parvovirus B19 IgG results using EIA and IFA analysis, were negative for both VP1 and VP2 by Western blot assay. Since the parvovirus B19 IgG EIA and IFA tests used were based on undenatured VP2 and VP1 antigens, respectively, it was suspected that a subpopulation of parvovirus B19 IgG-reactive specimens existed which react with neither denatured VP1 nor VP2 (i.e., linear epitopes). This, if true, would suggest that undenatured parvovirus B19 antigens are essential for the accurate detection of parvovirus B19 IgG.

Consequently, the primary aim of the study was to confirm the existence of a parvovirus B19 IgG EIA/IFApositive and Western blot-negative population. To this end, a panel of normal human sera was tested for parvovirus B19 IgG using the three diagnostic methods described and an alternative blot system that utilized undenatured VP2. Undenatured VP1 could not be included in this latter assay due to the solubilization steps necessary in purification from $S$. frugiperda cells. Secondly, it was decided to ascertain if a similar phenomenon was evident for parvovirus B19 IgM detection using Western blot analysis.

\section{MATERIALS AND METHODS Antigen Production}

Parvovirus B19 recombinant VP1 and VP2 capsid proteins were expressed in the baculovirus expression system for use in this study [Brown et al., 1990, 1991]. Recombinant VP1 infected cells were either utilized directly for slide preparation (immunofluorescence assay) or else extracted under denaturing conditions to produce purified VP1 protein for nitrocellulose strip preparation (Western/alternative blot). Insect cells expressing recombinant VP2 were lysed and the VP2 capsids purified under nondenaturing conditions for use in enzyme immunoassay or Western/alternative blot preparation.

Baculovirus expression of parvovirus B19 proteins. Recombinant baculoviruses (VP1 recombinant and VP2 recombinant) are each used to infect separately monolayers of Sf9 cells (ATCC CRL 1711) in TC100 medium containing $10 \%$ fetal calf serum [Brown et al., 1990].

VP1 purification. Infected Sf9 cells were harvested 3 days postinfection and lysed by sonication in phosphate-buffered saline. Cell debris and insoluble VP1 protein were pelleted by centrifugation at 3,000 g for $10 \mathrm{~min}$. After extensive washing with phosphate- buffered saline to remove soluble proteins, the insoluble VP1 protein was resuspended and dissolved in PBS/6-M guanidine-HCL and dialyzed with 6-M urea prior to SDS-PAGE [Laemmili et al., 1970] and electrotransfer [Towbin et al., 1979] to generate Western blot strips. This material was also used to prepare alternative blot strips using an airjet spray system (Airjet Model XY3000; Biodot, U.K.).

VP2 purification. Infected Sf9 cells were harvested 3 days postinfection and lysed by sonication in phosphate-buffered saline. Removal of cell debris was achieved by centrifugation at $3,000 \mathrm{~g}$ for $10 \mathrm{~min}$ and the resultant supernatant was layered onto a $15-30 \%$ (w/v) sucrose gradient and centrifuged for $3 \mathrm{hr}$ at 100,000 g. Pellets containing capsid VP2 were collected and used for enzyme immunoassay, alternative blot, and Western blot preparation.

\section{Immunoassay Formats}

A number of immunoassay formats were developed for the detection of parvovirus B19 antibodies. All the diagnostic kits used in this study and described below were obtained from Biotrin International (Mount Merrion, Dublin, Ireland).

Parvovirus B19 IgG enzyme immunoassay. The parvovirus B19 IgM enzyme immunoassay is a direct enzyme immunoassay for the detection of IgG-class antibodies to parvovirus B19 in human serum and plasma. Parvovirus B19 IgG, present in serum or plasma, diluted $1 / 100$, was bound to microwells that had been precoated with recombinant VP2 capsids. Following a wash step, horseradish peroxidase (HRP)labeled rabbit antihuman IgG was added, which bound to the IgG present. This complex was then detected by addition of tetramethyl benzidine (TMB) substrate. Sulfuric acid addition terminated the reaction after 10 min followed by absorbance measurement at $450 \mathrm{~nm}$. Specimens with an index value (specimen/cutoff absorbance ratio) greater than 1.1 were deemed positive.

Parvovirus B19 IgM enzyme immunoassay. The parvovirus B19 IgM enzyme immunoassay is a $\mu$-capture enzyme immunoassay for the detection of parvovirus B19 IgM in human serum and plasma (1/ 100 dilution). Microwells were precoated with rabbit antihuman IgM and total serum IgM was bound to the solid phase. Following a wash step, biotinylated parvovirus B19 VP2 capsids were added, which bound to any parvovirus B19 IgM present. After another wash step, streptavidin-peroxidase was added, which bound to the biotinylated VP2 present. This complex was then detected by addition of TMB substrate. Sulfuric acid addition terminated the reaction after 10 min followed by absorbance measurement at $450 \mathrm{~nm}$. Specimens with an index value (specimen/cutoff absorbance ratio) greater than 1.1 were deemed positive.

Parvovirus B19 IgG/IgM immunofluorescence assay. The parvovirus B19 immunofluorescence assay utilizes an indirect immunofluorescence antibody technique whereby the specimen is incubated with parvovirus B19 recombinant VP1 capsid antigen in insect 
TABLE I. Detection of Parvovirus B19 IgG by EIA, IFA, Western Blot (WB), and an Alternative Blot System (AB) in 108 Normal Human Sera

\begin{tabular}{lccccc}
\hline & & \multicolumn{5}{c}{ Parvovirus B19 IgG detection system } \\
\cline { 3 - 6 } Test result & Number (\%) & EIA $^{\mathrm{a}}$ & IFA $^{\mathrm{a}}$ & $\mathrm{WB}^{\mathrm{a}}$ & $\mathrm{AB}^{\mathrm{a}}$ \\
\hline Total positive & $75(69 \%)$ & & & & \\
Positive (Group 1) & 63 & + & + & $+^{\mathrm{b}}$ & + \\
Positive (Group 2) & 12 & + & + & - & $+^{\mathrm{c}}$ \\
Total negative & $33(31 \%)$ & - & - & - & - \\
\hline
\end{tabular}

aEIA: parvovirus B19 IgG enzyme immunoassay (utilizes undenatured VP2 antigen). IFA: parvovirus B19 IgG immunofluorescence assay (utilizes undenatured VP1 antigen). WB: parvovirus B19 IgG Western blot assay (utilizes denatured VP1 and VP2 antigens). AB: parvovirus B19 IgG alternative blot assay (utilizes denatured VP1 and undenatured VP2 antigen, respectively).

${ }^{\mathrm{b}}$ All 63 sera were reactive with VP1 only.

cAll 12 sera were reactive with VP2 only.

cells, previously stabilized on a glass slide [Brown et al., 1990]. The presence of parvovirus B19 antibodies in the sample led to the formation of a stable complex with VP1 antigen. Bound antibody was then reacted with a fluorescein-labeled antihuman IgG or IgM and the complex was visualized with the aid of a fluorescence microscope. Note that parvovirus B19 IgG was removed prior to parvovirus B19 IgM analysis.

Parvovirus B19 IgG/IgM Western blot. The Western blot immunoassay utilizes recombinant VP1 and VP2 viral antigens purified from baculovirus infected $S$. frugiperda cells that are electrotransfered onto nitrocellulose after SDS-PAGE. Any parvovirus B19 antibodies present in the human serum specimen bound to the immobilized antigens. After a wash step to remove any unbound antibody, HRP-conjugate (antihuman IgG or IgM) was added to the membrane. The bound antibody-conjugate complex was detected by the addition of the precipitating substrate diaminobenzidine tetrahydrochloride and $\mathrm{H}_{2} \mathrm{O}_{2}$. A positive result was indicated by the appearance of bands corresponding to the position of either or both VP1 and VP2 antigens. Note that parvovirus B19 IgG was removed prior to parvovirus B19 IgM analysis.

Parvovirus B19 IgG alternative blot system. This blot system utilizes purified recombinant VP1 and VP2 viral antigens obtained from infected $S$. frugiperda cells. Denatured VP1 antigen and undenatured VP2 capsids were sprayed separately as two bands onto nitrocellulose membrane using an airjet system. The assay procedure was identical to the Western blot Ig assay described above.

Comparison of EIA, IFA, and two blot methods for the detection of parvovirus B19 IgG in sera obtained from a blood donor population. Donor sera $(\mathrm{n}=108)$ were obtained from the Irish Blood Transfusion Service within 2 days of donation and were screened for parvovirus B19 IgG using the EIA based on undenatured VP2, IFA based on undenatured VP1, Western blot based on denatured VP1 and VP2, and the alternative blot format based on denatured VP1 and undenatured VP2 sprayed onto nitrocellulose without undergoing the denaturation inherent in electrophoresis.
Detection of parvovirus B19 IgM and IgG using Western blot assays in sera shown to be EIA- and IFA-positive for both antibody classes. Specimens ( $\mathrm{n}=85$ ) obtained from German clinical laboratories and which were shown to be positive by the aforementioned EIA and IFA test systems for both parvovirus B19 IgM and IgG, indicative of recent infection, were tested by Western blot. The parvovirus B19 IgG Western blot assays were performed to verify if parvovirus B19 IgG is reactive with both denatured proteins during such early convalescence and to investigate if any of these sera did not react with either or both denatured antigens.

Substantiation that denaturation of VP2 using the denaturing reagents used in VP1 purification would not have unexpected affects on parvovirus B19 IgM or IgG reactivity with VP2. VP2 purified following the usual VP2 nondenaturing purification procedure described earlier was denatured with guanidine hydrochloride and dialyzed with urea as per the VP1 solubilization method. Guanidine/urea-solubilized VP1 and VP2 was then used in IgG and IgM Western blots and the alternative IgG blot assay. The following four sample groups were tested, the first two for B19 IgM and IgG and the remaining two for B19 IgG only. The B19 IgM and IgG status of these samples had been previously determined by the Biotrin parvovirus B19 EIA, Western blot, and the alternative B19 IgG assays described previously. Group 1 includes B19 IgM/IgGnegative sera $(\mathrm{n}=5)$; Group 2, B19 IgM/IgG-positive sera $(\mathrm{n}=10)$; Group 3, B19 IgG-positive/IgM-negative sera $(n=5)$, which were VP1-reactive, VP2-unreactive on B19 IgG Western blots; Group 4, B19 IgG-positive/ IgM-negative sera $(n=5)$, which did not react with either VP1 or VP2 on B19 IgG Western blots.

\section{RESULTS}

The results for the comparison of the four detection methods for parvovirus B19 IgG in 108 blood donor sera are presented in Table I. This analysis indicates an overall parvovirus B19 IgG seropositivity rate of $69 \%$; in addition, identical serological results are obtained from the EIA, IFA, and alternative blot systems. However, 12 parvovirus B19 IgG-positive specimens, 
TABLE II. Parvovirus B19 IgM and IgG Positivity for 85 Specimens Tested by EIA, IFA, and Western Blot for the Respective Antibody Classes

\begin{tabular}{lccc}
\hline & \multicolumn{3}{c}{$\begin{array}{c}\text { Parvovirus B19 } \\
\text { detection system }\end{array}$} \\
\cline { 2 - 4 } Test result & EIA & IFA & Western blot $^{\text {a }}$ \\
\hline Parvovirus B19 IgM-positive & 85 & 85 & 85 \\
Parvovirus B19 IgG-positive & 85 & 85 & 85 \\
\hline
\end{tabular}

${ }^{a}$ EIA: parvovirus B19 IgG enzyme immunoassay. IFA: parvovirus B19 IgG immunofluorescence assay.

which represent $16 \%(12 / 75)$ of the confirmed positives, did not react to linear epitopes on either denatured VP1 or VP2 antigens when tested by Western blot.

The results for the comparison of the EIA, IFA, and Western blot methods for the detection of IgM and IgG in 85 sera are presented in Table II. All 85 sera contained both parvovirus B19 IgG and IgM as judged by all three test methods.

The reactivity incidence of parvovirus IgM and $\operatorname{IgG}$ with VP1 and VP2 in the Western blot assay for 85 sera is presented in Table III. Parvovirus B19 IgM reactivity against both VP1 and VP2 proteins was observed for all the specimens. However, upon testing for parvovirus B19 IgG only 69/85 (81\%) of specimens were reactive against both viral antigens. Of the remaining specimens, 16/85 (19\%) were reactive with VP1 only.

Examples of parvovirus B19 IgG results for sera tested by both Western blot and the alternative blot format are illustrated in Figures 1 and 2, respectively. There were four groups of result (A, B, C, and D) and three examples are shown for each. The parvovirus B19 IgG EIA and IFA result is also given for each Group (A-D). Group A individuals were reactive with VP1 and VP2 in both blot formats; these specimens were also parvovirus B19 IgM-positive. Group B individuals were VP1-positive in both blot formats but are only reactive with undenatured VP2 in the alternative blot format (Fig. 2). Group $\mathrm{C}$ individuals do not react with denatured VP1 and VP2 following Western blot analysis but reactive with undenatured VP2 in the alternative blot format. Group D are individuals who do not react with VP1 and VP2 in both blot systems and also when tested by parvovirus B19 IgG EIA and IFA. The banding pattern seen in Figure 1 for Group A and B specimens is due to parvovirus B19 IgG reactivity with degradation fragments of the VP1 protein. This pattern is not evident in the alternative blot format as the viral antigens are sprayed directly onto the nitrocellulose membrane.

The comparison of results between assays tested against undenatured VP2 and guanidine HCL/ureadenaturated VP2 is presented in Table IV. This denaturation made no difference to the results, except in the Group 3 sera. These Group 3 sera did not react with denatured VP2, when it was used in the alternative IgG blot system. This result was expected since these sera did not react with VP2 denatured with SDS-PAGE reagents by the original B19 IgG Western blot format.

\section{DISCUSSION}

This study has demonstrated for the first time that parvovirus B19 IgG from some individuals does not react against linear epitopes on both denatured parvovirus B19 VP1 and VP2 antigens. This finding is of significance as it clearly demonstrates the absolute requirement for the use of at least one undenatured antigen in immunodiagnostic tests designed for the detection of parvovirus B19 IgG. Moreover, it was demonstrated that either parvovirus B19 VP1 or VP2 capsid structure is sufficient for the attainment of maximum assay sensitivity given the high degree of correlation (100\%) between the different immunoassay formats.

From 108 sera that had been confirmed as parvovirus B19 IgG-positive by the alternative test methods, 12 did not react by Western blot (Table I). These sera were mostly strong positives by the other tests, thus eliminating the possibility of blot insensitivity as an explanation for the result. Furthermore, the antihuman IgG peroxidase conjugate used for both parvovirus B19 IgG Western blot and EIA was obtained from the same source, which confirms that differential conjugate reactivity is not responsible for the observed results. All 12 of these sera were detectable when nondenatured VP2 was used in the alternative blot format. Unfortunately, it was not possible to incorporate nondenatured VP1 into this format because denaturation is inherent to the VP1 purification process from baculovirus-infected insect cells. Western blots prepared from unpurified VP1, obtained by electrophoresis of infected $S$. frugiperda cell lysates, were tested with the unreactive parvovirus B19 IgG specimens $(\mathrm{n}=12)$. The results (not presented) were consistent with initial Western blot analysis whereby none of the 12 reacted against denatured VP1, which demonstrates that the observed lack of reactivity is not an artifact of the VP1 purification process. Finally, all 63 sera that were parvovirus B19 IgG-positive by Western Blot were reactive with VP1 only.

After identifying the occurrence of Western blot parvovirus B19 IgG nonreactivity, it was logical to investigate if there was a similar incidence with parvovirus B19 IgM detection using Western blot. The study of the 85 parvovirus B19 IgM EIA- and IFA-positive sera showed that all were detectable by a Western blot assay (Table II). In each case, both denatured antigens were reactive (Table III). These 85 parvovirus B19 IgM specimens had also been confirmed as parvovirus B19 IgG-positive and when assayed for parvovirus B19 IgG by Western blot all were reactive (Table II). Sixteen of these sera reacted with VP1 only and the remaining 69 reacted with both antigens (Table III).

The parvovirus B19 IgG Western blot results for the sera shown to be IgM-positive confirmed other investigators' observations that during an early postinfection period, the IgG is reactive with both denatured VP1 and VP2 in most cases [Söderlund et al., 1995]. It may be of significance that there were no Western blot IgG 
TABLE III. Parvovirus B19 IgM and IgG Western Blot Results Expressed in Terms of VP1 and VP2 Reactivity, Respectively for the 85 Specimens Presented in Table II

\begin{tabular}{lcccc}
\hline & \multicolumn{3}{c}{ Antigen reactivity pattern } \\
\cline { 2 - 5 } & $\begin{array}{c}\text { Number } \\
\text { VP1+/VP2+ } \\
(\%)\end{array}$ & $\begin{array}{c}\text { Number } \\
\text { VP1+/VP2- } \\
(\%)\end{array}$ & $\begin{array}{c}\text { Number } \\
\text { VP1-/VP2+ } \\
(\%)\end{array}$ & $\begin{array}{c}\text { Number } \\
\text { WP1-/VP2- } \\
(\%)\end{array}$ \\
\hline Parvovirus B19 IgM-positive & $\begin{array}{c}8(100 \%) \\
\text { Parvovirus B19 IgG-positive }\end{array}$ & $\begin{array}{c}0 \\
69\end{array}$ & 0 & 0 \\
\hline
\end{tabular}

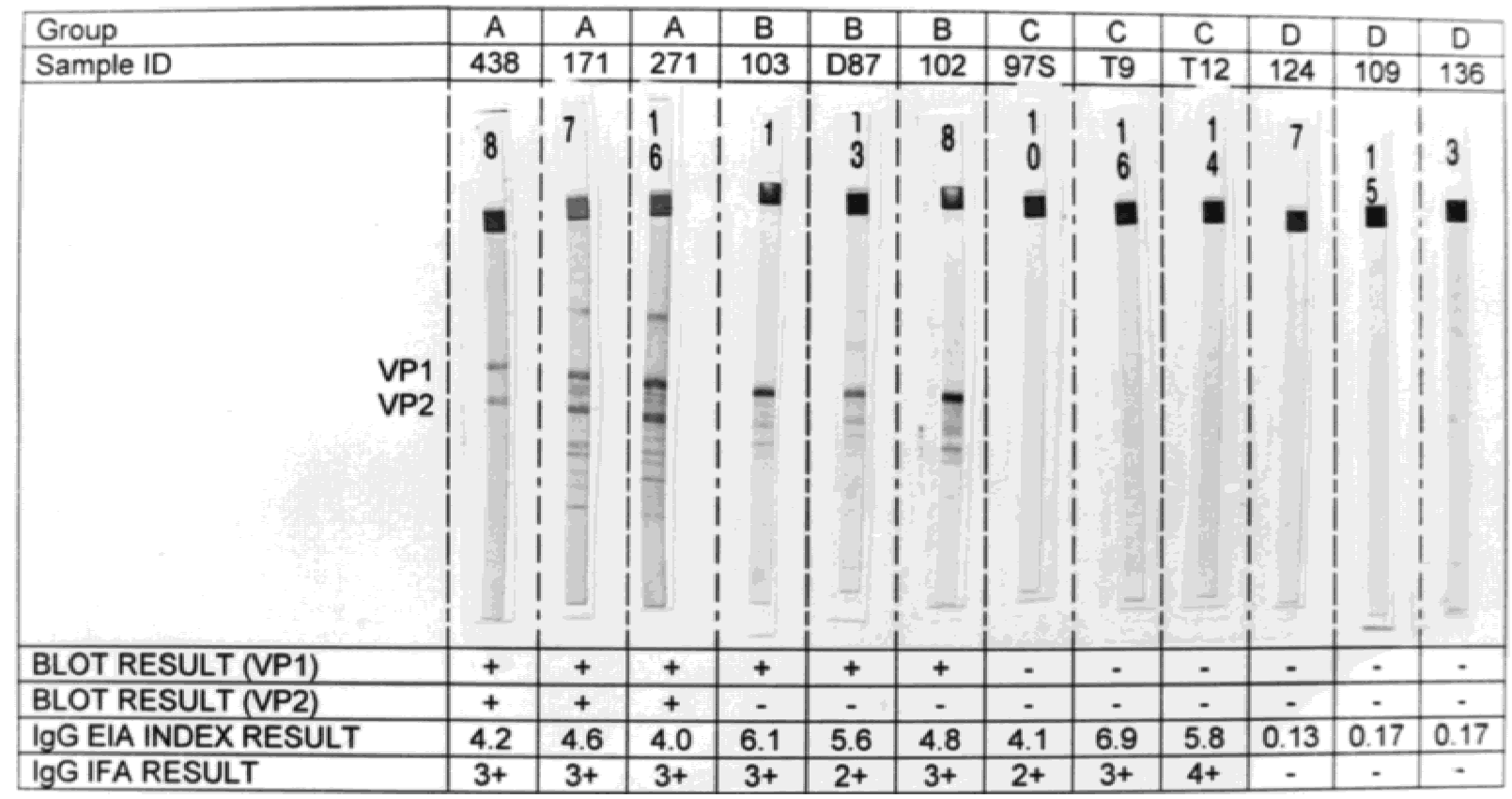

Fig. 1. Examples of results for sera tested for parvovirus B19 IgG using a Western blot that employs denatured VP1 and VP2 proteins for antibody capture. The parvovirus B19 IgG EIA and IFA result is also presented for each specimen. Group A: parvovirus B19 IgM- and IgG-positive specimens (Western blot-positive). Group B: parvovirus B19 IgG-positive specimens (Western blot-positive). Group C: parvovirus B19 IgG-positive specimens (Western blot-negative). Group D: parvovirus B19-negative specimens. Sample ID: unique specimen identification number. IgG index result: specimens with an index value (specimen/cutoff absorbance ratio) greater than 1.1 are deemed positive. Index value less than 0.9 implies negative result. IgG IFA result denotes fluorescence intensity: +, positive; $3+$ or $4+$, medium positive; $5+$, strong positive.

false negatives in this group, in contrast to those observed in the blood donor population tested. From these results it is possible to understand that the parvovirus B19 IgG nonreactivity with denatured VP2, and in some cases denatured VP1 and VP2, occurs after the early postinfection period. To substantiate this hypothesis, parvovirus B19 IgM and IgG seroconversion panels will have to be tested.

The study on the effect on B19 IgM or IgG reactivity with VP2 when it was denatured using the denaturing reagents used in VP1 purification (Table IV) showed that this denaturation had no significant affect on VP2 antigenicity, except as expected, when it was shown to eliminate VP2 reactivity with those B19 IgG-positive cases that also did not react with VP2 denatured by SDS-PAGE reagents by Western blot assays (Group 3 sera).

Söderlund et al. [1995] also compared parvovirus B19 IgG immunoreactivity between denatured and undenatured VP1. These investigators concluded that denaturation had little effect on VP1 antigenicity. How- ever, these conclusions were primarily obtained using serum pools and since the majority of parvovirus B19 IgG-positive specimens are reactive to denatured VP1, it is possible that they masked specimens lacking reactivity with linear epitopes of VP1. Furthermore, it may also be of significance that these previous studies used either the unique region of VP1 (as a $\beta$-galactosidase fusion protein) or a VP1/2 capsid combination, which may explain why Söderlund et al. [1995] did not make the observations regarding absence of reactivity against linear epitopes of VP1.

An interesting explanation was given by Söderlund et al. [1995] for the absence of parvovirus B19 IgG immunoreactivity with denatured VP2 in late convalescence. The hypothesis was that denatured VP2 has a "self" component and that after a transient breach of immunologic tolerance during early infection, immunoreactivity becomes confined to the VP1 unique region. The present study has shown that IgG reactivity toward linear epitopes on VP1 also undergoes temporal diminution. It would be tempting to speculate if the 


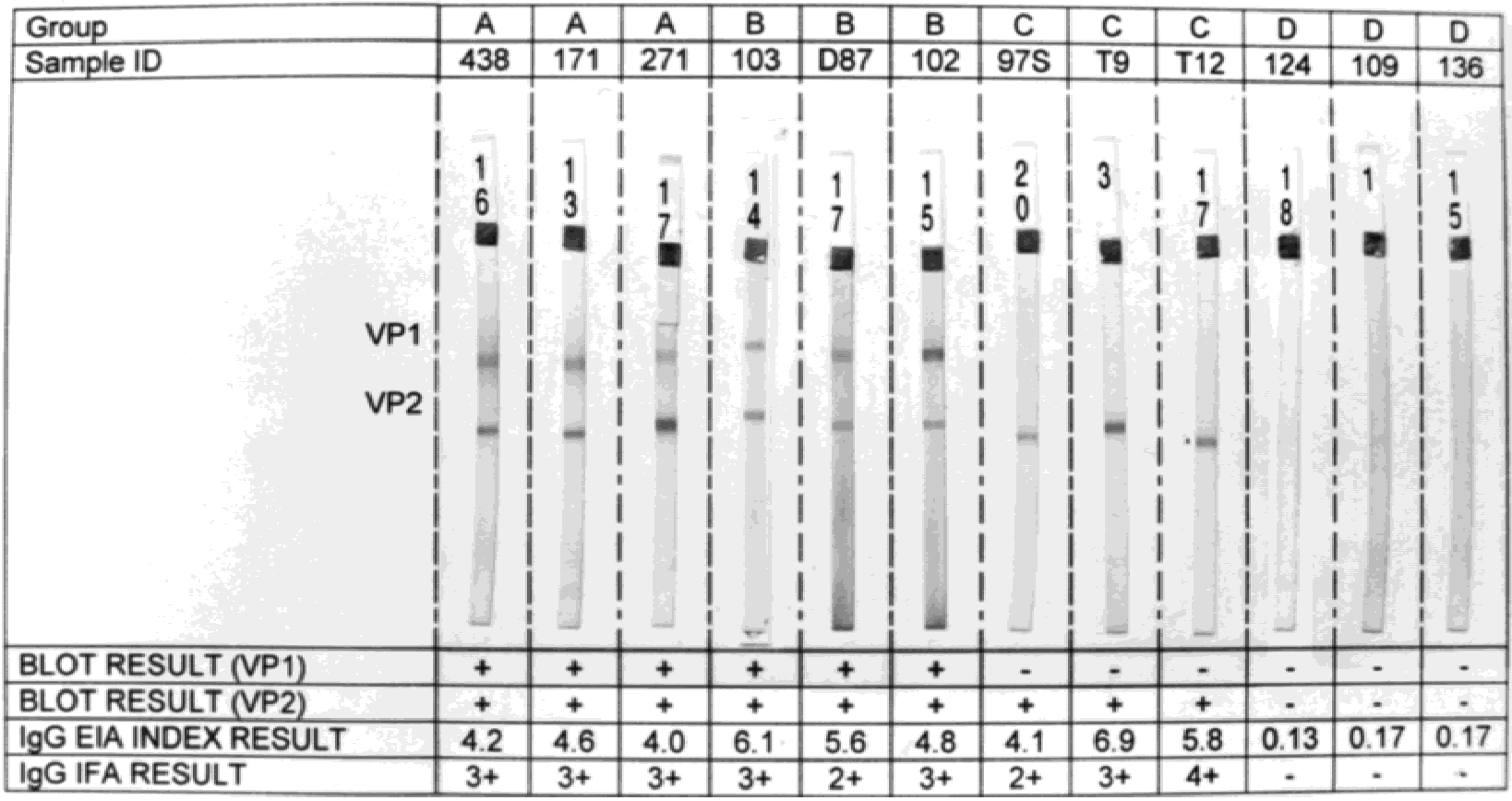

Fig. 2. Examples of results for sera tested for parvovirus B19 IgG using an alternative blot format that employs denatured VP1 and capsid VP2 protein for antibody capture. The parvovirus B19 IgG EIA and IFA result is also presented for each specimen. Group A: parvovirus B19 IgM- and IgG-positive specimens (Western blot-positive). Group B: parvovirus B19 IgG-positive specimens (Western blot-positive). Group C: parvovirus B19 IgG-positive specimens (Western blot-negative). Group D: parvovirus B19-negative specimens. Sample ID: unique specimen identification number. IgG index result: specimens with an index value (specimen/cutoff absorbance ratio) greater than 1.1 are deemed positive. Index value less than 0.9 implies negative result. IgG IFA result denotes fluorescence intensity: +, positive; $3+$ or $4+$, medium positive; $5+$, strong positive.

TABLE IV. Results of Parvovirus B19 IgM and IgG Reactivity With VP2 That Had Been Denatured Using VP1 Denaturation Reagents in Western Blot and Alternative IgG Blot Formats ${ }^{\text {a }}$

\begin{tabular}{|c|c|c|c|c|c|c|c|c|c|c|c|c|c|c|}
\hline \multirow[b]{3}{*}{ Serum group } & & & \multicolumn{4}{|c|}{ Western blot } & \multirow{2}{*}{\multicolumn{2}{|c|}{$\begin{array}{c}\begin{array}{c}\text { Alternative } \\
\text { blot }\end{array} \\
\text { IgG } \\
\end{array}$}} & \multicolumn{4}{|c|}{ Western blot } & \multirow{2}{*}{\multicolumn{2}{|c|}{$\begin{array}{l}\text { Alternative } \\
\text { IgG blot }\end{array}$}} \\
\hline & \multicolumn{2}{|c|}{ EIA } & \multicolumn{2}{|c|}{ IgM } & \multicolumn{2}{|c|}{ IgG } & & & \multicolumn{2}{|c|}{ IgM } & \multicolumn{2}{|c|}{ IgG } & & \\
\hline & $\overline{I g M}$ & $\operatorname{IgG}$ & VP1 & VP2 & VP1 & VP2 & $\overline{\mathrm{VP} 1}$ & VP2 & VP1 & $\mathrm{VP2}$ & $\mathrm{VP} 1$ & $\mathrm{VP2}$ & VP1 & VP2 \\
\hline Group 1 $(\mathrm{n}=5)$ & - & - & - & - & - & - & - & - & - & - & - & - & - & - \\
\hline Group $2(\mathrm{n}=10)$ & + & + & + & + & + & + & + & + & + & + & + & + & + & + \\
\hline Group $3(\mathrm{n}=5)$ & - & + & - & - & + & - & + & + & - & - & + & - & + & - \\
\hline Group $4(\mathrm{n}=5)$ & - & + & - & - & - & - & - & + & - & - & - & - & - & - \\
\hline
\end{tabular}

${ }^{a}$ Four serum groups were tested and a comparison made with results from EIA, Western blot, and the alternative IgG blot assays, which did not utilize Vp2 denatured with the VP1 denaturation reagents. Both Western blot and the alternative blot employed VP2 denatured using VP1 denaturing reagents.

unusual reactivity of parvovirus B19 IgG between linear and conformational epitopes is of medical significance, particularly in relation to autoimmune diseases to which the virus has been linked [Török, 1997].

\section{REFERENCES}

Anand A, Gray ES, Brown T, Clewley JP, Cohen BJ. 1987. Human parvovirus infection in pregnancy and hydrops fetalis. $\mathrm{N}$ Engl $J$ Med 316:183-186.

Anderson MJ, Jones SE, Fisher-Hoch SP, Lewis E, Hall SM, Bartlett CLR, Cohen BJ, Mortimer PP, Pereira MS. 1983. Human parvovirus, the cause of erythema infectiosum (fifth disease)? Lancet ii:1378.

Brown CS, Salimans MMM, Noteborn MHM, Harro TW. 1990. Antigenic parvovirus B19 coat proteins VP1 and VP2 produced in large quantities in a baculovirus expression system. Virus Res 15:197212

Brown CS, Van Lent JWM, Vlak JM, Spaan WJW. 1991. Assembly of empty capsids by using baculovirus recombinants expressing human parvovirus B19 structural proteins. J Virol 65:2702-2706.

Brown KE. 1997. Human parvovirus B19 epidemiology and clinical manifestations. In Anderson LJ, Young NS, editors. Human parvovirus B19. Basel: Karger, p 42-60.

Chipman PR, Agbandje-McKenna M, Kajigaya S, Brown KE, Young NS, Baker TS, Rossmann MG. 1996. Cryo-electron microscopy studies of empty capsids of human parvovirus B19 complexed with its cellular receptor. Proc Natl Acad Sci USA 93:7502-7506.

Franssila R, Söderlund M, Brown CS, Spaan WJM, Seppala I, Hedman K. 1996. IgG subclass response to human parvovirus B19 infection. Clin Diagn Virol 6:41-49.

Kurtzman GJ, Ozawa K, Cohen B, Hanson G, Oseas R, Blaese RM, Young NS. 1987. Chronic bone marrow failure due to persistant B19 parvovirus infection. N Engl J Med 317:287-294.

Laemmili UK. 1970. Cleavage of structural proteins during the assembly of the head of bacteriophage T4. Nature 227:680-685.

Lefrer JJ, Courouce AM, Bertrand Y, Girot R, Soulier JP. 1986. Hu- 
man parvovirus and aplastic crisis in chronic hemolytic anaemias: A study of 24 observations. Am J Hematol 23:271-275.

Morinet F, D'Auriol L, Tratschin JD, Galibert F. 1989. Expression of human parvovirus B19 protein fused to protein A in Escherichia coli: Recognition by IgM and IgG antibodies in human sera. J Gen Virol 70:3091-3097.

Ozawa K, Ayub J, Yu-Shu H, Kurtzman G, Shimada T, Young N. 1987. Novel transcription map for the B19 (human) pathogenic parvovirus. J Virol 61:2395-2406.

Serjeant GR, Serjeant GE, Thomas PW, Anderson MJ, Patou G, Pattison JR. 1993. Human parvovirus infection in homozygous sickle cell disease. Lancet 34i:1237-1240.

Söderlund M, Brown CS, Spaan WJ, Hedman L, Hedman K. 1995.
Epitope type-specific IgG responses to capsid proteins VP1 and VP2 of human parvovirus B19. J Inf Dis 172:1431-1436.

Towbin, H, Staehelin, T, Gordon J. 1979. Electrophoretic transfer of proteins from polyacrylamide gels to nitrocellulose sheets: Procedure and some applications. Proc Natl Acad Sci USA 76:43504354 .

Török TJ. 1997. Unusual clinical manifestations reported in patients with parvovirus B19 infection. In Anderson LJ, Young NS, editors. Human parvovirus B19. Basel: Karger, p 61-82.

Woolf AD, Campion GV, Chishick A, Wise S, Cohen BJ, Klouda PT, Caul O, Dieppe PA. 1989. Clinical manifestations of human parvovirus B19 in adults. Arch Intl Med 149:1153-1156. 